Pacific Journal of Mathematics

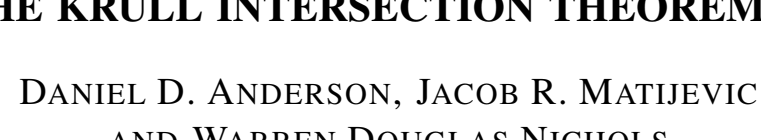




\title{
THE KRULL INTERSECTION THEOREM II
}

\author{
D. D. Anderson, J. Matijevic, and W. Nichols
}

Let $R$ be a commutative ring, $I$ an ideal in $R$ and $A$ an $R$-module. We always have $0 \subseteq\{a \in A \mid(1-i) a=0 \exists i$ $\in I\} \subseteq I \bigcap_{n=1}^{\infty} I^{n} A \subseteq \bigcap_{n=1}^{\infty} I^{n} A$. In this paper we inyestigate conditions under which certain of these containments may or may not be replaced by equality.

1. Introduction. This paper is a continuation of [1]. In $\S 2$ we show that for a nonminimal principal prime $(p), J=\bigcap_{n=1}^{\infty}(p)^{n}$ is a prime ideal and $p J=J$. An example is given to show that the condition that $(p)$ be nonminimal is necessary. We also consider the question of when a prime ideal minimal over a principal ideal has rank one. Of particular interest is the example of a domain $D$ with a doubly generated ideal $I$ such that $\bigcap_{n=1}^{\infty} I^{n} \neq I \bigcap_{n=1}^{\infty} I^{n}$. In $\S 3$ we prove that $\bigcap_{n=1}^{\infty} I^{n} A=$ $I \cap_{n=1}^{\infty} I^{n} A$ for any finitely generated module $A$ over a valuation ring. In $\$ 4$ we consider certain converses to the usual Krull Intersection Theorem for Noetherian rings. It is shown that for $(R, M)$ a quasi-local ring whose maximal ideal $M$ is finitely generated, many classical results for local rings are actually equivalent to the ring $R$ being Noetherian.

2. Some examples and counterexamples. In [1] we remarked that for a ring $R$ the following statements are equivalent: $\operatorname{dim} R=0$, (2) $\bigcap_{n=1}^{\infty} I^{n} A=I \bigcap_{n=1}^{\infty} I^{n} A$ for all finitely generated ideals $I$ and all $R$-modules $A$, (3) $\bigcap_{n=1}^{\infty} x^{n} A=x \bigcap_{n=1}^{\infty} x^{n} A$ for $x \in R$ and all $R$-modules $A$. This raises the question: For which ideals $I$ in a ring $R$ do we have $I \bigcap_{n=1}^{\infty} I^{n} A=\bigcap_{n=1}^{\infty} I^{n} A$ for all $R$-modules $A$ ? A modification of the example on page 11 of [1] yields

THEOREM 2.1. For a quasi-local ring $(R, M)$ and an ideal I the following statements are equivalent:

(1) $I^{n}=I^{n+1}$ for some $n$,

(2) for every $R$-module $A, I \bigcap_{n=1}^{\infty} I^{n} A=\bigcap_{n=1}^{\infty} I^{n} A$.

Proof. The implication (1) $\Rightarrow(2)$ is clear. Suppose that (2) holds but $I^{n} \supsetneqq I^{n+1}$ for all $n>0$. Choose $i_{n} \in I^{n}-I^{n+1}$. Let $F=$ $R x \oplus\left(\oplus \sum_{i=1}^{\infty} R y_{t}\right)$ be the free $R$-module on $\left\{x, y_{1}, y_{2}, \cdots\right\}$ and let $G$ be the sub-module of $F$ generated by the set $\left\{x-i_{1} y_{1}, x-i_{2} y_{2}, \cdots\right\}$ and let $A=F / G$. One can then verify that $I \bigcap_{n=1}^{\infty} I^{n} A \neq \bigcap_{n=1}^{\infty} I^{n} A$. 
It is well-known [7, page 74] that if $P$ is an invertible prime ideal in a domain, then $J=\bigcap_{n=1}^{\infty} P^{n}$ is a prime ideal, $J=P J$ and any prime ideal properly contained in $P$ is actually contained in $J$. We generalize this result. Recall that an ideal $I$ is finitely generated and locally principal if and only if it is a multiplication ideal (i.e., any ideal contained in $I$ is a multiple of $I$ ) and a weak-cancellation ideal (for two ideals $A$ and $B$, $A I \subseteq B I$ implies $A \subseteq B+(0: I)$ ). (For example, see [2] or [8].)

THEOREM 2.2. Let $R$ be a ring and $P$ a nonminimal finitely generated locally-principal prime ideal of $R$ and set $J=\bigcap_{n=1}^{\infty} P^{n}$. Then

(1) $J$ is prime,

(2) $P J=J$, and

(3) any prime ideal properly contained in $P$ is contained in $J$.

Proof. Let $a, b \in R \ni a, b \notin J$. We show that $a b \notin J$. Choose $n, m$ such that $a \in P^{n}-P^{n+1}$ and $b \in P^{m}-P^{m+1}$. Then since $P^{n}$ and $P^{m}$ are multiplication ideals, we get (a) $=P^{n} A_{1}$ and (b) $=P^{m} B_{1}$ where $A_{1} \not \subset P$ and $B_{1} \not \subset P$. Now (a)(b) $\subseteq P^{n+m+1}$ implies $A_{1} B_{1} P^{n+m} \subseteq P^{n+m+1}$. Since $P^{n+m}$ is a weak-cancellation ideal, $A_{1} B_{1} \subseteq P+\left(0: P^{n+m}\right)$. Let $Q \subsetneq P$ be a prime ideal, then $\left(0: P^{n+m}\right) P^{n+m}=0 \subsetneq Q$ gives $\left(0: P^{n+m}\right) \subseteq Q \subsetneq P$ and hence $A_{1} B_{1} \subseteq P$. Thus $A_{1}$ or $B_{1} \subseteq P$, a contradiction. Hence $J$ is prime. Let $j \in J$, then $j \in P$ so $(\mathrm{j})=P A$. Since $P$ is a nonminimal prime, $P \not \subset J$, hence $A \subseteq J$, so $j \in P J$. For (3), let $Q$ be a prime ideal properly contained in $P$ and let $q \in Q$. Then $(q)=P Q_{1} \subseteq Q$ and $P \not \subset Q$ implies $Q_{1} \subseteq Q \subset P$. Continuing we get $(q) \subseteq J$.

COROllary 2.3. Let $(p)$ be a nonminimal principal prime ideal. Then $J=\bigcap_{n=1}^{\infty}(p)^{n}$ is prime, $p J=J$ and prime ideal $Q \subsetneq(p)$ is contained in $J$.

The above corollary is false if $(p)$ is a minimal prime ideal. For example, in $Z /(4) \bigcap_{n=1}^{\infty}(\overline{2})^{n}$ is not prime. However, in this example condition (2) still holds. In the following example we show that condition (2) may also fail.

EXAmPle 2.4. Let $k$ be a field and let $R=k\left[X, Z, Y_{1}, Y_{2}, \cdots\right]$ be the polynomial ring over $k$ in indeterminants $X, Z, Y_{1}, Y_{2}, \cdots$. Let $A=\left(X-Z Y_{1}, X-Z^{2} Y_{2}, X-Z^{3} Y_{3}, \cdots\right)$ and put $\bar{R}=R / A$. Then $(X, Z)$ is a prime ideal of $R$ minimal over $A$ and hence $(\overline{X, Z})$ is a minimal prime ideal of $\bar{R}$ (- denotes passage to $\bar{R}$ ). Moreover, $(\overline{X, Z})=(\bar{Z})$, so $(\bar{Z})$ is a minimal principal prime ideal of $\overline{\bar{R}}$. However, $\bigcap_{n=1}^{\infty}(\bar{Z})^{n} \neq(\bar{Z}) \bigcap_{n=1}^{\infty}(\bar{Z})$ because $\bar{X} \in \bigcap_{n=1}^{\infty}(\bar{Z})^{n}$ but $\bar{X} \notin(\bar{Z}) \bigcap_{n=1}^{\infty}(\bar{Z})^{n}$.

The Principal Ideal Theorem states that a prime ideal in a Noetherian domain minimal over a principal ideal has rank one. In general 
a prime ideal minimal over a principal ideal need not have rank one. In fact, a principal prime (p) has rank one if and only if $\bigcap_{n=1}^{\infty}(p)^{n}=0$. More generally, if $P$ is a rank one prime, any $a \in P$ must satisfy $\bigcap_{n=1}^{\infty}(a)^{n}=0$ (see Corollary 1.4 [9] or Theorem 1 [1]). This raises the question: In a domain, does a prime $P$ minimal over a principal ideal (a) with $\bigcap_{n=1}^{\infty}(a)^{n}=0$ imply that rank $P=1$ ? This question is answered in the negative by Example 5.2 [9]. Finally we ask the question: In a domain, does a finitely generated prime $P$ satisfying $\bigcap_{n=1}^{\infty} P^{n}=0$, minimal over a principal ideal, have rank 1? While we are not able to answer this question, we do show that there can not be "too many" primes below $P$.

THEOREM 2.5. Let $R$ be a domain and let $P$ be a finitely generated prime ideal minimal over a principal ideal $R x$. Then rank $P=1$ if and only if $\cap\{Q \in \operatorname{Spec}(R) \mid Q$ is directly below $P\}=0$.

Proof. The implication $(\Rightarrow)$ is clear. Conversely, let $\left\{Q_{\alpha}\right\}$ be the set of prime ideals directly below $P$ (this set is nonempty by Zorn's Lemma). The hypothesis of the theorem is preserved by passage to $R_{p}$, so we may assume that $R$ is quasi-local. Thus $(R, P)$ is quasi-local, $P$ is finitely generated, and $R x$ is $P$-primary. By Theorem $1[1], \bigcap_{n=1}^{\infty} P^{n} \subseteq$ $\cap\{Q \mid Q$ directly below $P\}=0$. Let $(\hat{R}, \hat{P})$ be the $P$-adic completion of $R$. Then $(\hat{R}, \hat{P})$ is a complete (Noetherian) local ring. Now $\hat{R} x$ is still $\hat{P}$-primary, so by the Principal Ideal Theorem, $\operatorname{dim} \hat{R} \leqq 1 . \quad$ If $\operatorname{dim} \hat{R}=0$, then $\hat{P}^{n}=0$ for some $n$ and hence $P^{n}=0$. This contradiction shows that $\operatorname{dim} \hat{R}=1$. Let $P_{1}, \cdots, P_{n}$ be the minimal primes of $\hat{R}$ and let $Q_{1}=P_{1} \cap R$. Now $\cap\{Q \mid Q$ directly below $P\}=0$ implies that there exist infinitely many primes directly below $P$. Hence $\exists y \in Q_{0}-\bigcup_{t=1}^{n} Q_{i}$ where $Q_{0}$ is a prime directly below $P$. Now $\hat{R} y \notin \cup_{i=1}^{n} P_{\iota}$, so $\hat{R} y$ is $\hat{P}$-primary. Hence $\hat{R} y \cap R$ is $P$-primary. But by Theorem $1[1]$ we see that $Q_{0}$ is closed in the $P$-adic topology, and hence $\hat{R} y \cap R \subseteq Q_{0}$. This is a contradiction because $\hat{R} y \cap R$ is $P$-primary.

The proof of Theorem 2.5 does yield the following result. Let $P$ be a finitely generated prime ideal in a domain minimal over a principal ideal. Then rank $P=1$ if and only if $\bigcap_{n=1}^{\infty} P_{P}^{n}=0$ (or equivalently, if $\bigcap_{n=1}^{\infty} P^{(n)}=0$ where $P^{(n)}$ is the $n$-symbolic power of $P$ ).

We end this section with an example of a domain $D$ and a doubly generated ideal $I$ in $D$ satisfying $I \bigcap_{n=1}^{\infty} I^{n} \neq \bigcap_{n=1}^{\infty} I^{n}$. This is the best possible counterexample as $\bigcap_{n=1}^{\infty}(x)^{n}=(x) \bigcap_{n=1}^{\infty}(x)^{n}$ for all principal ideals in a domain.

EXAMPLE 2.6. Let $k$ be a field, $S=k\left[W, W^{\frac{1}{2}}, W^{\frac{1}{3}}, W^{\frac{1}{5}}, \cdots\right]$, and $R_{0}=S\left[X, U_{2}, U_{3}, U_{5}, U_{7}, \cdots\right]$. Then $R_{0}[Y, 1 / Y]$ is a graded domain, 
with degree $R_{0}=0$, degree $Y=1$ and degree $1 / Y=-1$. Let $R$ be the graded subdomain $R_{0}\left[Y,\left(W^{\frac{1}{2}}-X U_{2}\right) / Y,\left(W^{\frac{1}{3}}-X U_{3}\right) / Y, \cdots\right]$. Then $I=$ $(X, Y)$ is a homogeneous ideal of $R$. Put $J=\bigcap_{n=1}^{\infty} I^{n}$ so that $J$ is also a homogeneous ideal. We show that $J \neq I J$.

Write $Z_{p}=W^{1 / p}-X U_{p}$. Then $R_{0}=k\left[W, Z_{2}, Z_{3}, Z_{5}, \cdots, X, U_{2}, U_{3}\right.$, $\left.U_{5}, \cdots\right]$. We have the relation $\left(Z_{p}+X U_{p}\right)^{p}=W$ and hence

$$
Z_{p}^{p}=W-X^{p} U_{p}^{p}-\left(\begin{array}{l}
p \\
1
\end{array}\right) Z_{p} X^{p-1} U_{p}^{p-1}-\cdots-\left(\begin{array}{c}
p \\
p-1
\end{array}\right) Z_{p}^{p-1} X U_{p}
$$

Note that $R_{0}$ is spanned as a $k$-vector space by the monomials $Z_{p_{1}}^{e_{1}} \cdots Z_{p_{r}}^{e_{r}} W^{n_{0}} X^{n_{1}} U_{q_{1}}^{f_{1}} \cdots U_{q_{s}}^{f_{s}}$, where $0<e_{t}<p_{i}$. We show that these monomials are $k$-independent, and thus form a $k$-basis. To see this, define the degree of the monomial $W^{e_{1} / p_{1}+\cdots+e_{r} / p_{r}+n_{0}} X^{n_{1}} U_{q_{1}}^{f_{1}} \cdots U_{q_{s}}^{f_{s}}$ $\left(0<e_{\imath}<p_{\imath}\right)$ to be $\left(e_{1} / p_{1}+\cdots+e_{r} / p_{r}+n_{0}, n_{1}, 0, \cdots, 0, f_{1}, 0, \cdots, 0, f_{s}, 0 \cdots\right)$ where $f_{t}$ appears in the $s_{t}$-th position after $n_{1}$ if $q_{t}$ is the $s_{t}$-th prime. Order the degrees lexicographically. Then define the degree of a polynomial to be the degree of the largest term. We find that the degree of $Z_{p_{1}}^{e_{1}} \cdots Z_{p_{r}}^{e_{r}} \cdots Z_{p_{r}}^{e_{r}} W^{n_{0}} X^{n_{1}} U_{q_{1}}^{f_{1}} \cdots U_{q_{s}}^{f_{s}}\left(0<e_{t}<p_{i}\right)$ to be $\left(e_{1} / p_{1}+\right.$ $\left.\cdots+e_{r} / p_{r}+n_{0}, n_{1}, 0, \cdots, f_{1}, \cdots, f_{s}, 0, \cdots\right)$ as above. Each such monomial has a different degree, and hence these monomials are $k$ independent. Let us write $T=k\left[X, W, U_{2}, U_{3}, Y_{5}, \cdots\right]$. We see that $R_{0}=T \oplus R_{0_{z}}$ as a $T$-module, where $R_{0 z}$ is generated as a $T$-module by the $Z_{p_{1}}^{e_{1}} \cdots Z_{p_{r}}^{e_{r}}, 0<e_{t}<p_{t}, r \geqq 1$. Let $H$ be the ideal of $R_{0}$ generated by the $Z_{p}$ 's. Since $H \supset R_{0 z}$, we have $H=(H \cap T) \oplus R_{0 z}$ as a $T$ module. Now

$$
\begin{aligned}
{\left[I^{m}\right]_{0}=\left[(X, Y)^{m}\right]_{0} } & =X^{m} R_{0}+X^{m-1} Y R_{-1}+\cdots+Y^{m} R_{-m} \\
& =X^{m} R_{0}+X^{m-1} H+\cdots X H^{m}=(X, H)^{m}
\end{aligned}
$$

as an ideal of $R_{0}$. Notice that since $W=\left(Z_{p}+X U_{p}\right)^{p}$, we have $W \in(X, H)^{m}$ for all $m$. Now $H \cap T$ is generated as a $T$-module by the $W-X^{p} U_{p}^{p}$. Thus $(X, H)$ is generated by $X, W$, and the $Z_{p_{1}}^{e_{1}} \cdots Z_{p_{r}}^{e_{r}}$ $(r \geqq 1)$ and $(X, H)^{m}$ is generated by $X^{m}, W$, the $Z_{p_{1}}^{e_{1}} \cdots Z_{p}^{e_{r}} W(r \geqq 1)$, and the $Z_{p_{1}}^{e_{1}} \cdots Z_{p_{r}}^{e_{r}} X^{n_{0}}$ with $e_{1}+\cdots+e_{r}+n_{0} \geqq m$. It follows that $J_{0}=$ $\bigcap_{m=1}^{\infty}(X, H)^{m}=W R_{0}$.

We claim that $W \notin[I J]_{0}=X J_{0}+Y J_{-1}$. In fact, we claim that $W \notin X J_{0}+Y R_{-1}=X W R_{0}+H$. Since $H \supset R_{0 z}$, the ideal

$$
\left(X W R_{0}+H\right) \cap T=\left(X W, W-X^{2} U_{2}^{2}, W-X^{3} U_{3}^{3}, W-X^{5} U_{5}^{5}, \cdots\right) .
$$

Suppose that $W \in X J_{0}+Y R_{-1}$, then 


$$
W=a X W+b_{2}\left(W-X^{2} U_{2}^{2}\right)+\cdots+b_{p}\left(W-X^{p} U_{p}^{p}\right)
$$

$a, b_{\imath} \in T$. Write $b_{i}=c_{i}+\lambda_{t}$ where $\lambda_{i} \in k$ and $c_{\imath} \in T$ with no constant term. Cancelling $W$, we get

$$
\lambda_{2} X^{2} U_{2}^{2}+\cdots+\lambda_{p} X^{p} U_{p}^{p}=a X W-c_{2} X^{2} U_{2}^{2}-\cdots-c_{p} X^{p} U_{p}^{p}
$$

But this is a contradiction since none of the terms on the left appear on the right.

3. Valuation rings. We call a ring $R$ a valuation ring if any two ideals of $R$ are comparable. In Theorem 2 [1] we proved that for $R$ a Prüfer domain, $I$ an ideal in $R$ and $A$ a torsion-free $R$-module, $I \bigcap_{n=1}^{\infty} I^{n} A=\bigcap_{n=1}^{\infty} I^{n} A$. In this section we prove that for $R$ a valuation ring, $I$ an ideal in $R$ and $A$ a finitely generated $R$-module, $I \bigcap_{n=1}^{\infty} I^{n} A=$ $\bigcap_{n=1}^{\infty} I^{n} A$. We begin with the ring case.

THEOREM 3.1. Let $V$ be a valuation ring and I a nonzero ideal in $V$. Then exactly one of the following occurs:

(1) $I=I^{2}$ is prime,

(2) $I^{n} \supsetneqq I^{n+1}$ for all $n, \bigcap_{n=1}^{\infty} I^{n}$ is a prime ideal in $V$, and $\bigcap_{n=1}^{\infty} I^{n}=$ $\bigcap_{n=1}^{\infty}(i)^{n}$ for any $i \in I-I^{2}$. In particular, $\bigcap_{n=1}^{\infty} I^{n}=I \bigcap_{n=1}^{\infty} I^{n}$.

(3) $I^{n}=0$ for some $n$.

Proof. First suppose that $I=I^{2}$ and let $a b \in I$. Suppose that $a, b \notin I$, so that $I \subsetneq(a)$ and $I \subsetneq(b)$. Hence $I=I^{2} \subseteq(a)(b) \subseteq I$ so $I=$ $(a b)$. Thus $I=I^{2}$ implies $I=0$, a contradiction. Next suppose that $I \neq I^{2}$, but $I^{n} \supsetneqq I^{n+1}=I^{n+2}$. Let $i \in I^{n}-I^{n+1}$. Then for $m>1, I^{n+1}=$ $I^{m n} \supseteq(i)^{m} \supseteq I^{m(n+1)}=I^{n+1}$, in particular $(i)^{2}=(i)^{3}$, so $(i)^{2}=0$. Hence $0=(i)^{2} \supseteq I^{2(n+1)}=I^{n+1}$. Finally, suppose that $I^{n} \supsetneqq I^{n+1}$ for all $n$. For $i \in I-I^{2}, I \supseteq(i) \supseteq I^{2}$, so that $I^{n} \supseteq(i)^{n} \supseteq I^{2 n}$ and hence $\bigcap_{n=1}^{\infty} I^{n}=$ $\bigcap_{n=1}^{\infty}(i)^{n}$. Suppose that $x y \in \bigcap_{n=1}^{\infty} I^{n}$. If $x, y \notin \bigcap_{n=1}^{\infty} I^{n}$, then there exist integers $s$ and $t$ such that $I^{s} \subsetneq(x)$ and $I^{t} \subsetneq(y)$. Hence $I^{s+t} \subseteq(x y) \subseteq$ $\bigcap_{n=1}^{\infty} I^{n}$ so $I^{s+t}=I^{s+i+1}$. This contradiction shows that $\bigcap_{n=1}^{\infty} I^{n}$ must be prime. Suppose that $x \in \bigcap_{n=1}^{\infty} I^{n}$. Then $x=s i^{2}$ for some $s \in V$ and $i \in I$. Hence si or $i \in \bigcap_{n=1}^{\infty} I^{n}$ because $\bigcap_{n=1}^{\infty} I^{n}$ is prime. Thus $\bigcap_{n=1}^{\infty} I^{n}=I \bigcap_{n=1}^{\infty} I^{n}$.

THeOrem 3.2. Let $V$ be a valuation ring, $I$ an ideal in $V$ and $A$ a finitely generated $V$-module. Then $\bigcap_{n=1}^{\infty} I^{n} A=I \bigcap_{n=1}^{\infty} I^{n} A$.

Proof. By the previous theorem we are reduced to the case where $I=(i)$ is a principal ideal and $\bigcap_{n=1}^{\infty}(i)^{n}$ is prime. Put $B=\left(\bigcap_{n=1}^{\infty}(i)^{n}\right) A$, so that $B \subseteq \bigcap_{n=1}^{\infty}(i)^{n} A$. It suffices to show that $\bigcap_{n=1}^{\infty}(i)^{n}(A / B)=$ 
(i) $\bigcap_{n=1}^{\infty}(i)^{n}(A / B)$. But as $\operatorname{ann}(A / B) \supseteq \bigcap_{n=1}^{\infty}(i)^{n}$, we may assume that $\bigcap_{n=0}^{\infty}(i)^{n}=0$, so that $V$ is a valuation domain. Let $A=V a_{1}+\cdots+V a_{s}$ and assume that ann $\left(a_{1}\right) \supseteq \cdots \supseteq$ ann $\left(a_{s}\right)$. We may assume that $(i)^{n} \supseteq$ ann $\left(a_{1}\right)$ (for otherwise $i^{n} a_{1}=0$ for large $n$ and hence we may assume that $\left.A=V a_{2}+\cdots+V a_{s}\right)$. Thus $0=\bigcap_{n=1}^{\infty}(i)^{n} \supseteq$ ann $\left(a_{1}\right)$, so that $A$ is actually torsion-free. The result now follows from Lemma 1 [1].

4. "Almost" Noetherian rings. Let $R$ be a Noetherian ring, $I$ an ideal in $R$, and $A$ a finitely generated $R$-module. One version of the Krull Intersection Theorem states that $\bigcap_{n=1}^{\infty} I^{n} A=$ $\{x \in A \mid(1-i) x=0 \exists i \in I\}$. In fact, by Theorem 3 [1] this holds for $R$ locally Noetherian and $A$ locally finitely generated. In this section we consider to what extent the converse is true. We begin with the quasi-local case.

THEOREM 4.1. Let $(R, M)$ be a quasi-local ring whose maximal ideal $M$ is finitely generated. Then the following statements are equivalent :

(1) $R$ is Noetherian,

(2) $\bigcap_{n=1}^{\infty} M^{n} N=0$ for all finitely generated $R$-modules $N$,

(3) every finitely generated ideal of $R$ has a primary decomposition,

(4) for finitely generated ideals $A$ and $B$ of $R$, there exists an integer $n$ such that $\left(A+B^{l}\right) \cap\left(A: B^{l}\right)=A$ for $l \geqq n$,

(5) $\bigcap_{n=1}^{\infty}\left(M^{n}+A\right)=A$ for all finitely generated ideals $A$ of $R$,

(6) $B=A+M B$ with $A$ a finitely generated ideal of $R$ implies $A=B$.

Proof. The implications (1) $\Rightarrow(2)$ and (1) $\Rightarrow(3)$ are well known. Assume that (3) holds and let $A$ and $B$ be finitely generated ideals. Suppose that $A=Q_{1} \cap \cdots \cap Q_{m}$ where $Q_{i}$ is $P_{i}$-primary. Assume that $B \subseteq P_{1}$ precisely for $i>k$. For $i \leqq k,\left(Q_{i}: B^{n}\right) B^{n} \subseteq Q_{1}$ and $B^{n} \not \subset P_{\imath}$ implies $\left(Q_{\imath}: B^{n}\right)=Q_{\imath}$ for all $n$. For $i>k$, there exists an integer $n_{t}$ such that $B^{n_{i}} \subseteq Q_{\imath}$ because $B$ is finitely generated. Set $n=$ $\max \left\{n_{i}\right\}$. Then for $l \geqq n, \quad A: B^{l}=Q_{1} \cap \cdots \cap Q_{k}$ and $A+B^{l} \subseteq$ $Q_{k+1} \cap \cdots \cap Q_{m}$. Hence $A \subseteq\left(A: B^{l}\right) \cap\left(A+B^{l}\right) \subseteq Q_{1} \cap \cdots \cap Q_{m}=$ $A$. Next we show that (4) implies (5). Let $A$ be a finitely generated ideal of $R$. Clearly $A \subseteq \cap_{n=1}^{\infty}\left(M^{n}+A\right)$. Suppose that $x \in$ $\bigcap_{n=1}^{\infty}\left(M^{n}+A\right)$. Then by (4) $A+(x) M=\left(A+(x) M+M^{k}\right)$ $\cap\left((A+(x) M): M^{k}\right)$ for large $k$. But $x \in A+M^{k}$ so $A+(x) M=$ $A+(x)$. Thus $x \in A$ by Nakayama's Lemma. Setting $N=R / A$ we see that (2) implies (5). As (6) holds in any (Noetherian) local ring, it remains to prove $(5) \Rightarrow(1)$ and $(6) \Rightarrow(1)$. Suppose that $R$ is not Noetherian. Then there exists an ideal $P \neq M$ maximal with respect to not being finitely generated and $P$ is necessarily prime. Let $z \in M-P$. 
Then $P+(z)$ is finitely generated, say by $p_{1}+r_{1} z, \cdots, p_{n}+r_{n} z$ where $p_{1}, \cdots, p_{n} \in P$. We claim that $P=\left(p_{1}, \cdots, p_{n}\right)$. Let $p \in P \subseteq P+(z)$, so that

$$
\begin{aligned}
p & =a_{1}\left(p_{1}+r_{1} z\right)+\cdots+a_{n}\left(p_{n}+r_{n} z\right)= \\
& =a_{1} p_{1}+\cdots+a_{n} p_{n}+\left(a_{1} r_{1}+\cdots+a_{n} r_{n}\right) z .
\end{aligned}
$$

Since $P$ is a prime ideal and $z \notin P, a_{1} r_{1}+\cdots+a_{n} r_{n} \in P$. Hence $P=$ $\left(p_{1}, \cdots, p_{n}\right)+P z=\left(p_{1}, \cdots, p_{n}\right)+P^{n} Z^{n}$ for $n \geqq 1$. Thus either (5) or (6) implies that $P=\left(p_{1}, \cdots, p_{n}\right)$.

It is necessary to assume that $M$ is finitely generated as is seen by the example $R=k\left[\left\{X_{l}\right\}_{i=1}^{\infty}\right] /\left(\left\{x_{l}\right\}_{l=1}^{\infty}\right)^{2}$ where $k\left[\left\{x_{l}\right\}_{i=1}^{\infty}\right]$ is the polynomial ring over the field $k$ in countably-many indeterminates. If we replace the quasi-local ring $(R, M)$ with a quasi-semilocal ring $\left(R, M_{1}, \cdots, M_{n}\right)$ where $M_{1}, \cdots, M_{n}$ are finitely generated and replace $M$ with $J=M_{1} \cap \cdots \cap M_{n}$, then Theorem 4.1 remains true. The equivalence of (1) and (5) is a slight generalization of Exercise 4 [5, page 246]. Condition (4) has been studied in [4].

COROLlaRY 4.2. For a ring $R$ the following statements are equivalent:

(1) $R$ is locally Noetherian,

(2) $\bigcap_{n=1}^{\infty}\left(M^{n}+A\right)=\{r \in R \mid(1-m) r \in A \exists m \in M\}$ for all finitely generated ideals $A$ of $R$ and all maximal ideals $M$ of $R$, and for every maximal ideal $M$ of $R, M_{M}$ is a finitely generated ideal in $R_{M}$.

Proof. (1) $\Rightarrow(2)$. The first statement follows from Theorem 3 [1] applied to the ring $R / A$ which is locally Noetherian. The second statement is obvious. (2) $\Rightarrow(1)$. Follows from the previous theorem.

THEOREM 4.3. For a ring $R$ the following conditions are equivalent:

(1) $R$ is Noetherian,

(2) the maximal ideals of $R$ are finitely generated and every finitely generated ideal of $R$ has a primary decomposition.

Proof. That $(1) \Rightarrow(2)$ is well-known. Therefore we may assume that $R$ satisfies (2). It follows from Theorem 4.1 that $R$ is locally Noetherian. Theorem 1.4 [3] gives that $R$ is Noetherian.

The results of this section raise the question: Is a locally Noetherian ring whose maximal ideals are finitely generated necessarily Noetherian? The answer is no.

EXAMPLE 4.4. The ring $R=Z[\{x / p \mid p$ a prime $\}]$ is two dimen- 
sional, integrally closed, locally Noetherian with all maximal ideals finitely generated, but $R$ is not Noetherian. In fact, $R$ is not even a Krull domain.

This ring is given in [6] as an example of a locally polynomial ring over $Z$ which is not a polynomial ring over $Z$. We wish to thank Professor R. Gilmer for pointing out this example to us. ${ }^{1}$

First, the ring $R$ is not Noetherian because the ideal $(\{x / p \mid p$ a prime $\}$ ) is not finitely generated. The maximal ideals of $R$ have the form $(p, f(x / p))$ where $p \in Z$ is prime and $f(x / p)$ is an irreducible polynomial (in $x / p$ ) mod $p$. The remaining statements follow from the fact that $R$ localized at a maximal ideal $M$ (with $M \cap Z=(p)$ ) is a localization of the polynomial ring $Z_{(p)}[x / p]$ at $M_{Z-(p)}$.

\section{REFERENCES}

1. D. D. Anderson, The Krull Intersection Theorem, Pacific J. Math., 57 (1975), 11-14.

2. - Multiplication ideals, multiplication rings, and the ring $R(X)$, Canad. J. Math. 28 (1976), $760-768$.

3. J. Arnold and J. Brewer, Commutative rings which are locally Noetherian, J. Math. Kyoto Univ., 11-1 (1971), 45-49.

4. W. E. Barnes and W. M. Cunnea, Ideal decompositions in Noetherian rings, Canad. J. Math., 17 (1965), 178-184.

5. N. Bourbaki, Commutative Algebra, Addison-Wesley, Reading, Mass., 1972.

6. P. Eakins and J. Silver, Rings which are almost polynomial rings, Trans. Amer. Math. Soc., 174 (1972), 425-449.

7. R. Gilmer, Multiplicative Ideal Theory, Marcel Dekker, New York, 1972.

8. P. J. McCarthy, Principal elements of lattices of ideals, Proc. Amer. Math. Soc., 30 (1971), 43-45.

9. J. Ohm, Some counterexamples related to integral closure in $D[[X]]$, Trans. Amer. Math. Soc., 122 (1966), 321-333.

Received January 28, 1976.

ViRginia Polytechnic Institute and State University

UNIVERSITY OF SOUTHERN CALIFORNIA

AND

PenNSylvania State University

Current address: UNIVERSITY OF MisSOURI-COLUMBIA

${ }^{1}$ This example is due to P. Eakin, R. Gilmer, and W. Heinzer. 



\section{Pacific Journal of Mathematics}

\section{Vol. 66, No. $1 \quad$ November, 1976}

Helen Elizabeth. Adams, Factorization-prime ideals in integral domains ............ Patrick Robert Ahern and Robert Bruce Schneider, The boundary behavior of Henkin's kernel.

Daniel D. Anderson, Jacob R. Matijevic and Warren Douglas Nichols, The Krull

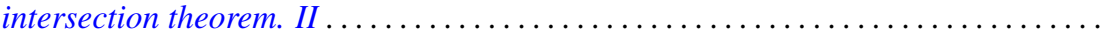

Efraim Pacillas Armendariz, On semiprime P.I.-algebras over commutative regular

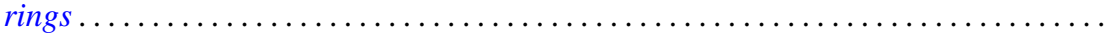

Robert H. Bird and Charles John Parry, Integral bases for bicyclic biquadratic fields

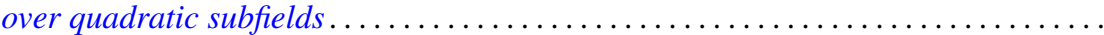

Tae Ho Choe and Young Hee Hong, Extensions of completely regular ordered

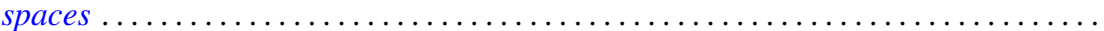

John Dauns, Generalized monoform and quasi injective modules ...............

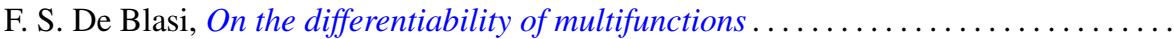

Paul M. Eakin, Jr. and Avinash Madhav Sathaye, R-endomorphisms of $R[[X]]$ are

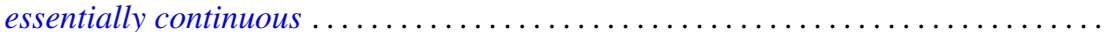

Larry Quin Eifler, Open mapping theorems for probability measures on metric

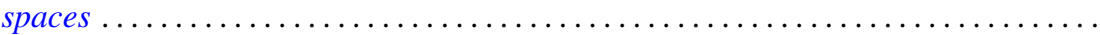

Garret J. Etgen and James Pawlowski, Oscillation criteria for second order self adjoint

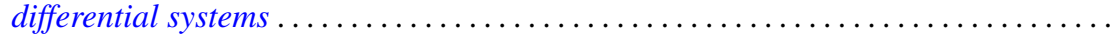

Ronald Fintushel, Local $S^{1}$ actions on 3-manifolds .

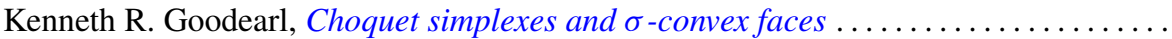

John R. Graef, Some nonoscillation criteria for higher order nonlinear differential

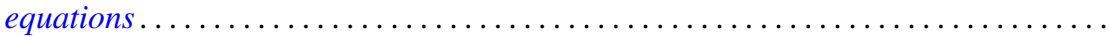

Charles Henry Heiberg, Norms of powers of absolutely convergent Fourier series: an example.

Les Andrew Karlovitz, Existence of fixed points of nonexpansive mappings in a space

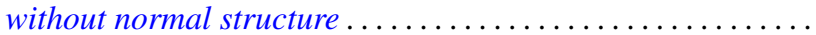

Gangaram S. Ladde, Systems of functional differential inequalities and functional

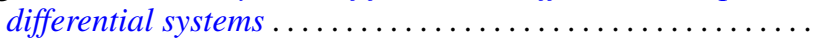

Joseph Michael Lambert, Conditions for simultaneous approximation and interpolation with norm preservation in $C[a, b]$.

Ernest Paul Lane, Insertion of a continuous function.

Robert F. Lax, Weierstrass points of products of Riemann surfaces .

Dan McCord, An estimate of the Nielsen number and an example concerning the Lefschetz fixed point theorem...

Paul Milnes and John Sydney Pym, Counterexample in the theory of continuous functions on topological groups...

Peter Johanna I. M. De Paepe, Homomorphism spaces of algebras of holomorphic functions

Judith Ann Palagallo, A representation of additive functionals on $L^{p}$-spaces,

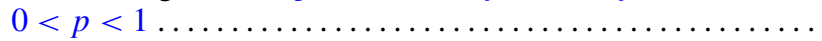

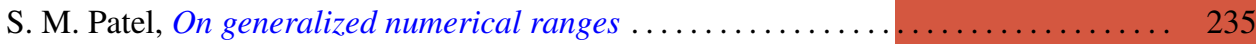

Thomas Thornton Read, A limit-point criterion for expressions with oscillatory

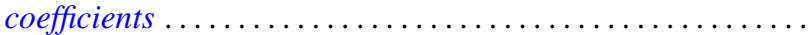

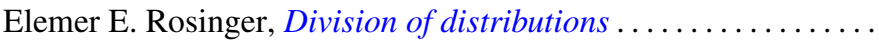

Peter S. Shoenfeld, Highly proximal and generalized almost finite

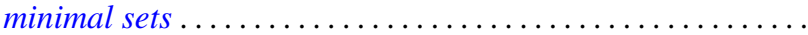

R. Sirois-Dumais and Stephen Willard, Quotient-universal sequential spaces

Robert Charles Thompson, Convex and concave functions of singular values of matrix sums....

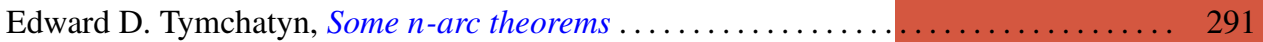

